\title{
Reflectometry measurements during operation of the dynamic ergodic divertor at TEXTOR
}

\author{
A. Krämer-Flecken, S. Soldatov ${ }^{1}$, C. Busch, Y. Liang, \\ M. von Hellermann ${ }^{2}$, R. Wolf, O. Zimmermann and the \\ TEXTOR-Team
}

\author{
Institut für Plasmaphysik, Forschungszentrum Jülich GmbH, EURATOM Association, 52425 \\ Jülich, Germany* \\ ${ }^{1}$ Nuclear Fusion Institute, Kurchatov Institute, 123128 Moscow, Russia \\ ${ }^{2}$ FOM Institute for Plasma Physics Rijnhuizen, Association EURATOM-FOM, PO Box \\ 1207, 3430 BE Nieuwegein, The Netherlands \\ E-mail: a.kraemer-flecken@fz-juelich.de
}

Received 5 October 2005, accepted for publication 10 May 2006

Published 17 August 2006

Online at stacks.iop.org/NF/46/S730

\begin{abstract}
The O-mode correlation reflectometer at TEXTOR is used for the investigation of the propagation and the properties of the turbulence at the plasma edge. The multi-horn antenna set-up allows the investigation of the correlation properties of the turbulence and its rotation. Turbulence and plasma rotation for different plasma conditions are found to be in agreement and based on this comparison the radial electric field is estimated. In addition the density fluctuation level is estimated from the standard deviation of the phase of the reflected signal.

The influence of a magnetic perturbation field, generated by the dynamic ergodic divertor (DED), on the turbulence and transport properties is studied and compared with plasmas without such a field perturbation. The strength and radial range of the perturbation field can be widely varied. Together with tangential neutral beam injection in coand counter-current directions, the turbulent transport is investigated. The combination of neutral beam injection and the DED enables the modification of the plasma rotation profile. It can lead either to the generation of a locked mode or the formation of a transport barrier.
\end{abstract}

PACS numbers: 52.35.Ra, 52.55.Fa, 52.55.Tn, 52.70.Gw

(Some figures in this article are in colour only in the electronic version)

\section{Introduction}

The investigation of turbulence phenomena and its properties, as well as finding methods to influence and reduce turbulence, are of major interest because they influence the transport properties and stability of a thermonuclear plasma. For this purpose it is necessary to measure the turbulence properties for different plasma parameters to learn how to influence the turbulence in a way that is beneficial for the confinement.

First observations of a reduction in the broad band (BB) turbulence, as well as in quasi-coherent (QC) modes $(80 \leqslant f \leqslant 180 \mathrm{kHz}$ ) were made at $\mathrm{T}-10$ [1] during the temporary transition from saturated to improved ohmic confinement. However, a simple relationship between the turbulence reduction and the diffusion coefficient could not be deduced. Also, during the generation of transport barriers,

\footnotetext{
* Partner in the trilateral Euregio Cluster.
}

a reduction in the turbulence level $[2,3]$ caused by an increased $E \times B$ velocity shear was observed. The increased velocity shear is due to a bifurcation of the radial electric field $E_{r}$. A correlation between long-wavelength, turbulence driven density fluctuations and particle transport was reported from TFTR [4]. More recent studies of the turbulence spectrum show that QC, high m-number modes evolve during the generation/annihilation of a barrier. However, they are strongly suppressed as long as the barrier is developed [5]. This supports the assumption that the property of the QC mode is closely related to particle transport. Recently, a reduction in turbulence in pellet-injected improved confinement plasmas was reported [6]. All these results demonstrate that turbulence reduction is related to a decrease in transport and an increase in confinement.

This paper reports on the measurement of turbulence properties at the plasma edge during dynamic ergodic divertor 


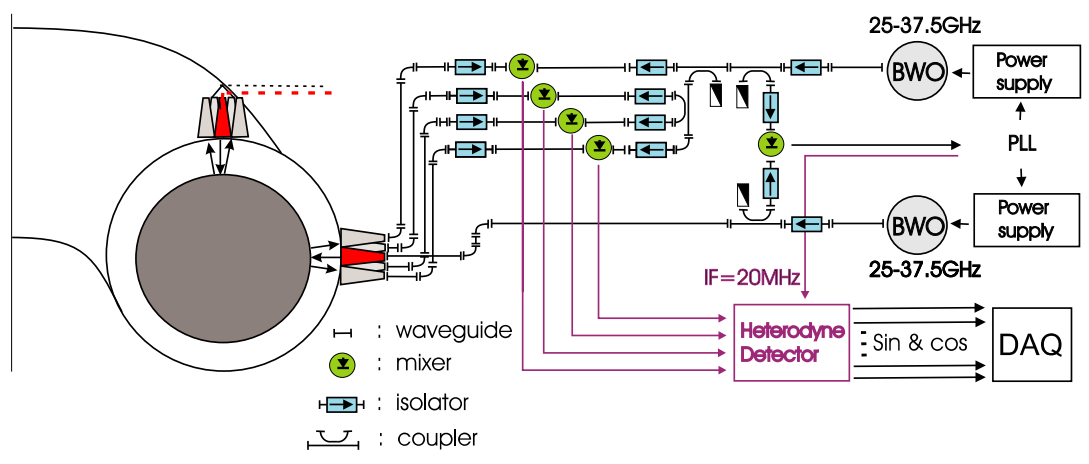

Figure 1. A sketch of the heterodyne O-mode reflectometry system operated at TEXTOR. The system operates with two antenna arrays, one in the equatorial plane and one on top of the vessel. Short and long poloidal correlations can be investigated.

(DED) operation. In the following section the reflectometry system and the DED are explained. The next section describes the experimental plasma conditions and the change in global plasma parameters during DED operation. Section 4 describes the analysis of the reflectometer data with emphasis placed on the comparison between turbulence rotation and plasma rotation as discussed in section 4.2. The experimental results for different scenarios are discussed in section 5. The achieved results and conclusions are summarized in section 6.

\section{Reflectometry and the DED at TEXTOR}

An O-mode poloidal correlation reflectometry system [7] is installed at TEXTOR $\left(R_{0}=1.75 \mathrm{~m}, a=0.47 \mathrm{~m}\right)$. Its relatively low frequency (LF) range of $26 \leqslant f \leqslant 37 \mathrm{GHz}$, $\left(0.8 \times 10^{19} \leqslant n_{c} \leqslant 1.7 \times 10^{19} \mathrm{~m}^{-3}\right.$, where $n_{c}$ is the cutoff density) is adjusted along the requirements of the DED operation in plasmas with medium electron density. The reflectometer can be operated with two antenna arrays-one in the equatorial plane and the other on top of the vessel with each consisting of 5 pyramidal horn antennas. Both arrays are installed in the same poloidal cross section. The configuration of the system with the antenna arrays is shown in figure 1. For the reported experiments only the midplane antennae system is used. The antenna array in the equatorial plane is arranged with three antennas in one column. This column is aligned symmetrically about the equatorial plane. In the toroidal direction, next to the first column a second column with two antennas is installed, also symmetrically aligned. All antennas of this equatorial array are focused on $R_{0}=1.75 \mathrm{~m}$. According to the antenna geometry the sequence of angle distance is $\Delta \Theta_{1,2,3,4}=[0.024 ; 0.0489 ; 0.0733 ; 0.0977]$ radians. For the antennas and TEXTOR geometries the sensitivity in $k_{\Theta}$ is restricted to $k_{\Theta} \leqslant 4 \mathrm{~cm}^{-1}$ [7]. The system provides the density fluctuation level as well as spectral and correlation measurements. Short distance correlation measurements between arbitrary antennas of one array (top or midplane) are possible. Long distance correlation measurements between an arbitrary antenna pair from the top and the equatorial plane can also be performed. The signal for each antenna is measured with a quadrature detector. The sine and cosine component from the quadrature detectors are sampled at a rate of $1 \mathrm{MHz}$ for $t \leqslant 4 \mathrm{~s}$.

At TEXTOR an external helical magnetic field $[8,9]$ is generated by the DED and can be applied for a wide range of plasma conditions. For the generation of the magnetic perturbation field a set of 16 coils are wrapped helically around the high field side (HFS) of the vessel. The perturbation field matches the field line structure of the $q=3$ surface. Depending on the phase of the neighbouring coils, the system can be operated in the so called $3 / 1,6 / 2$ and $12 / 4$ configurations. The penetration depth of the perturbation is largest in the $3 / 1$ configuration and smallest in the $12 / 4$ configuration. The current in the coils is variable; $I_{\mathrm{DED}} \leqslant$ $3.75 \mathrm{kA}$ in the $3 / 1$ configuration and $I_{\mathrm{DED}} \leqslant 15 \mathrm{kA}$ in the $12 / 4$ configuration. Furthermore, the DED can be operated in $\mathrm{dc}$ as well as ac with frequencies up to $10 \mathrm{kHz}$. A detailed technical description can be found in [10].

The DED causes an ergodization of the plasma edge by generating there island chains. Such a concept is closely related to island divertors in stellarators. The topology of the divertor depends on the distance of the resonant surface from the DED coils, which can be adjusted by the plasma current and/or the toroidal magnetic field. The choice of the safety factor can cause either a large stochastic region and a small laminar region, with a small connection length to the wall, or large laminar regions with only small ergodic layers. The main purpose of the stochastic field is the control of impurity and edge transport. In addition, the DED can be used to control MHD activities and toroidal rotation. For a detailed investigation on these topics the reader is referred to [11,12].

As shown at Tore Supra [13] the onset of the ergodic divertor modifies the radial electric field at the plasma edge and moves the inversion point deeper into the plasma. Therefore, the radial impurity influx is reduced (impurity screening).

\section{Investigated plasma scenarios}

TEXTOR is equipped with two tangential neutral beam lines each with 1.3 MW maximum power, injecting in the co- and counter-current directions respectively. The power injected into the plasma is controlled by an aperture [14]. Ohmic plasmas at TEXTOR rotate in counter-current direction and $P_{\mathrm{NBI}} \approx 300 \mathrm{~kW}$ in the co-current direction is needed to compensate the ohmic plasma rotation. This offers the possibility of studying the DED effects for different toroidal plasma rotation profiles. The experiments reported here are performed for the 3/1 and 12/4 DED configurations with dc operation only and with different amounts of tangential neutral beam heating. The plasma parameters (plasma current $I_{\mathrm{p}}$, 
Table 1. Main plasma parameters used in the two different DED configurations.

\begin{tabular}{llllllllll}
\hline Scenario & \multicolumn{7}{c}{ Plasma parameter } & & \multicolumn{2}{c}{ DED parameter } \\
\cline { 2 - 4 } & $I_{p} / \mathrm{kA}$ & $B_{T} / \mathrm{T}$ & $q_{a}$ & $\Delta_{\text {horz }} / \mathrm{m}$ & $P_{\mathrm{Co}} / \mathrm{kW}$ & $P_{\text {Counter }} / \mathrm{kW}$ & & $I_{\text {DED }} / \mathrm{kA}$ & Config. \\
\hline 1 & 300 & 2.25 & 4.8 & 0.04 & 280 & 0 & & 2.0 & $3 / 1$ \\
2 & 300 & 2.25 & 4.8 & 0.04 & 280 & 1300 & & 2.5 & $3 / 1$ \\
3 & 370 & 1.9 & 3.0 & -0.05 & 800 & 0 & & $10-12.5$ & $12 / 4$ \\
\hline
\end{tabular}

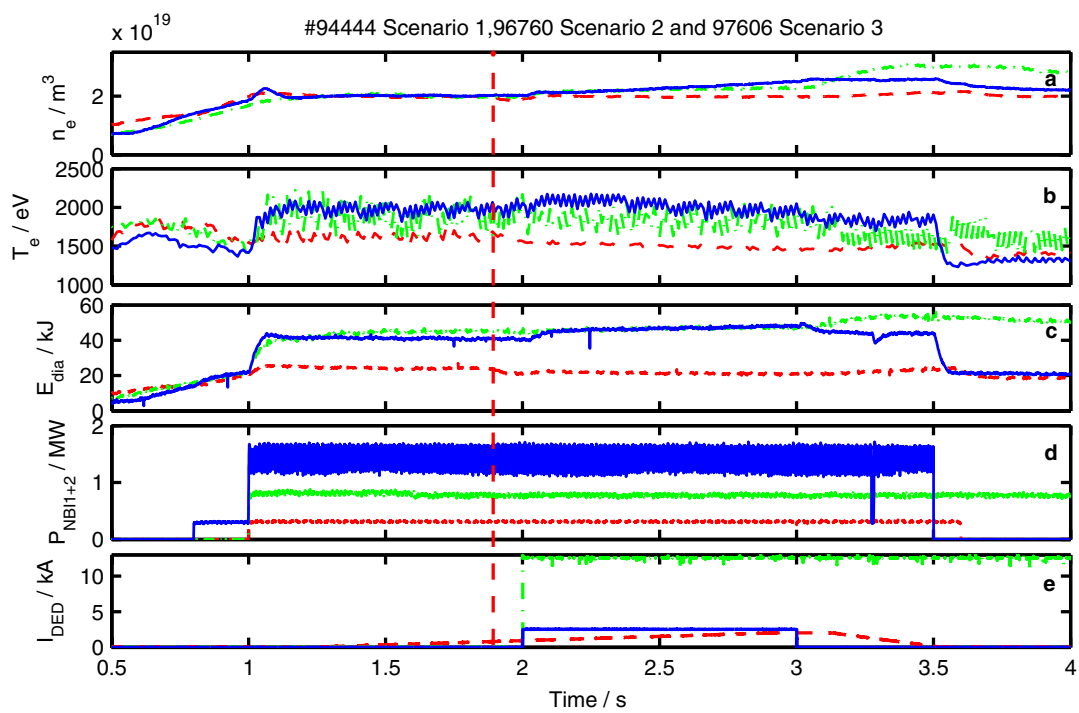

Figure 2. Overview of several plasma parameters with applied DED. Red curves belong to Scenario 1, blue curves to Scenario 2 (both with the $3 / 1$ configuration) and green curves to Scenario 3 with the 12/4 configuration of the DED. The subplots show: $(a)$ line averaged density; (b) central electron temperature; $(c)$ diamagnetic energy; $(d)$ input power of the neutral beam injection lines and $(e)$ the DED current. The vertical dashed line marks the onset of the locked 2/1 mode in Scenario 1.

magnetic field $B_{\mathrm{T}}$ and horizontal position $\Delta_{\text {horz }}$ ) are different in both DED configurations. An overview of the plasma scenarios is given in table 1. A detailed analysis of the global results of different DED configurations on MHD stability, plasma rotation and transport can be found in [11]. For all plasma scenarios a full frequency scan of the reflectometry system is performed on a shot-to-shot basis. The line averaged electron density is in the range $2.0 \cdot 10^{19} \leqslant \overline{n_{e}} \leqslant 2.5 \cdot 10^{19} \mathrm{~m}^{-3}$. In both configurations the DED was operated during the flat top of the discharge with $I_{\mathrm{DED}}=2.0 \mathrm{kA}$ in the $3 / 1$ configuration and $10.0 \leqslant I_{\mathrm{DED}} \leqslant 12.5 \mathrm{kA}$ in the $12 / 4$ configuration.

\subsection{Scenario 1}

Figure 2 shows the line averaged density $\left(\bar{n}_{e}\right)$, central electron temperature $\left(T_{e}\right)$, diamagnetic energy $\left(E_{\mathrm{dia}}\right)$, neutral beam power $\left(P_{\mathrm{NBI}}\right)$ of both neutral beam injectors and the DED current for different scenarios with DED operation. The dashed curve shows a plasma with the DED in the $3 / 1$ configuration and $P_{\mathrm{NBI}}=280 \mathrm{~kW}$ in the co-current direction. Note that at $t=1.8 \mathrm{~s}$, at a certain threshold of $I_{\mathrm{DED}} \geqslant 0.7 \mathrm{kA}$, a drop in $\bar{n}_{e}$ is observed and correlates with a decrease in $T_{e}$ and $E_{\mathrm{dia}}$ of $15 \%$. This observation is caused by a locked $m / n=2 / 1$ mode, centered at $r / a \approx 0.64$ and generated by the DED [15]. Using electron cyclotron emission (ECE) and soft $\mathrm{X}$-ray radiation (SXR) [16] diagnostics the width of the locked mode is deduced to be $w \approx 0.06 \mathrm{~m}$. The orientation of the mode within the vessel can be assigned using two ECE measurements, $110^{\circ}$ toroidally apart. Based on this observation, one of the X-points of the locked mode is close to the location of the reflectometry equatorial antenna array. One reason for the onset of the large island is the flat rotation profile [17], measured by charge exchange recombination spectroscopy (CXRS), as shown in figure 3. It shows a flat profile from the centre to $r / a=0.65$, the position of the $2 / 1$ island.

\subsection{Scenario 2}

The solid curve in figure 2 displays the case for neutral beam injection predominately in the counter-current direction with $P_{\mathrm{NBI}}^{\mathrm{Counter}}=1.3 \mathrm{MW}$ plus $P_{\mathrm{NBI}}^{\mathrm{Co}}=0.3 \mathrm{MW}$ in the cocurrent direction. The co-current beam is needed for CXRS measurements. Except for the toroidal rotation direction and the injected power all other plasma parameters are the same as in Scenario 1. With the onset of the DED, $\bar{n}_{e}$ increases and continues to do so throughout the whole DED phase. $E_{\mathrm{dia}}$ also increases (by 10-15\%) and this improvement remains during the DED period. Although a slight increase of $T_{e}$ is seen at the beginning of the DED phase, the temperature drops overall, due to the increasing electron density. The transition back to L-mode confinement is observed with the end of the DED operation. The analysis of the toroidal rotation profiles (see solid lines in figure 3) show a constant decrease for countercurrent rotation during the DED period compared with the profile without DED. A difference of $\Delta \Omega_{\Phi} \approx 15 \mathrm{krad} \mathrm{s}^{-1}$ is observed at $r / a \leqslant 0.65$. 


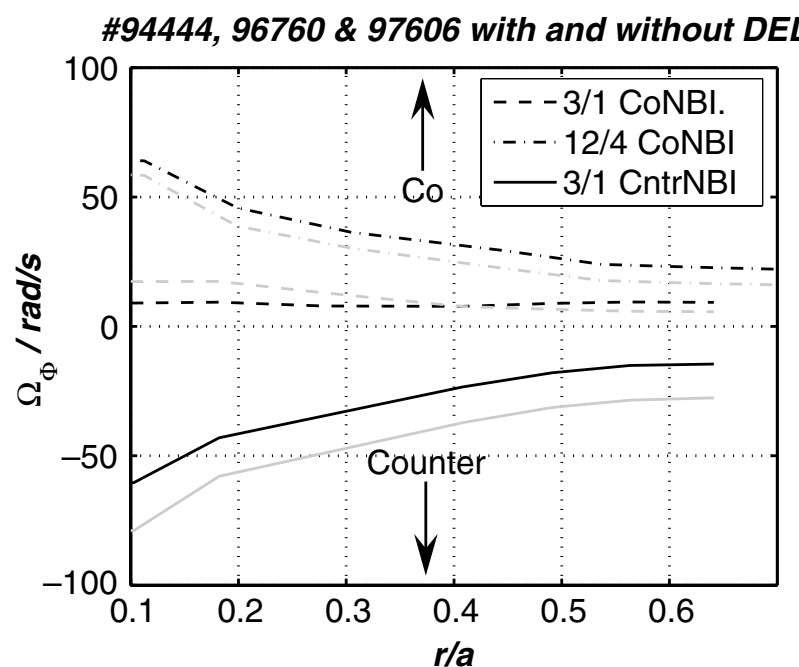

Figure 3. Rotation profile for three discharge types. For each type the profile without (gray curve) and with the DED (black curve) is shown. The flat profile belongs to co-injection with the 3/1 DED (Scenario 1). The negative profile belongs to the counter-injection with the 3/1 DED (Scenario 2) and the positive profiles to the 12/4 DED with co-injection (Scenario 3).

\subsection{Scenario 3}

The last scenario uses the DED in the 12/4 configuration with $P_{\mathrm{NBI}}=800 \mathrm{~kW}$ in the co-current direction. This scenario was optimized for divertor operation. The safety factor $q_{a}=3.0$ is chosen and generates a large laminar region, where the transport properties are comparable to the scrape off layer in a poloidal divertor [18]. For a detailed discussion of the divertor properties the reader is referred to the following papers $[19,20]$. To enlarge the DED effects at the plasma edge the plasma is shifted by $5 \mathrm{~cm}$ towards the HFS. In this so-called ergodic divertor operation [19] a decrease in $T_{e}$ is observed for a narrow layer $\Delta r=0.03 \mathrm{~m}$ at the plasma edge due to the generation of a laminar zone. Due to the operation of the DED the magnetic flux tubes at the boundary have a relatively short connection length. Since the laminar zone is a helically wrapped flux tube, the position of the reflectometry with respect to the laminar zone is important. In our case, calculation with the ATLAScode [21] shows that the antenna sight line looks through the laminar zone. Concerning central plasma quantities no significant effects are observed (see figure 2) despite a small increase in the central toroidal rotation (see dash dotted line in figure 3). The change in $\Omega_{\phi}$, in the co-current direction with DED in the plasma centre is a general observation and is discussed in [12].

\section{Analysis of reflectometer data}

\subsection{Methodology and approaches for data analysis}

To investigate the turbulence characteristics spectral (frequency domain) and correlation (time domain) analyses of the experimental data are performed. The spectral analysis includes amplitude (or power), cross-phase and coherence spectra calculations and gives important information on the mode of fluctuations contributing to the measured signal. Figure 4
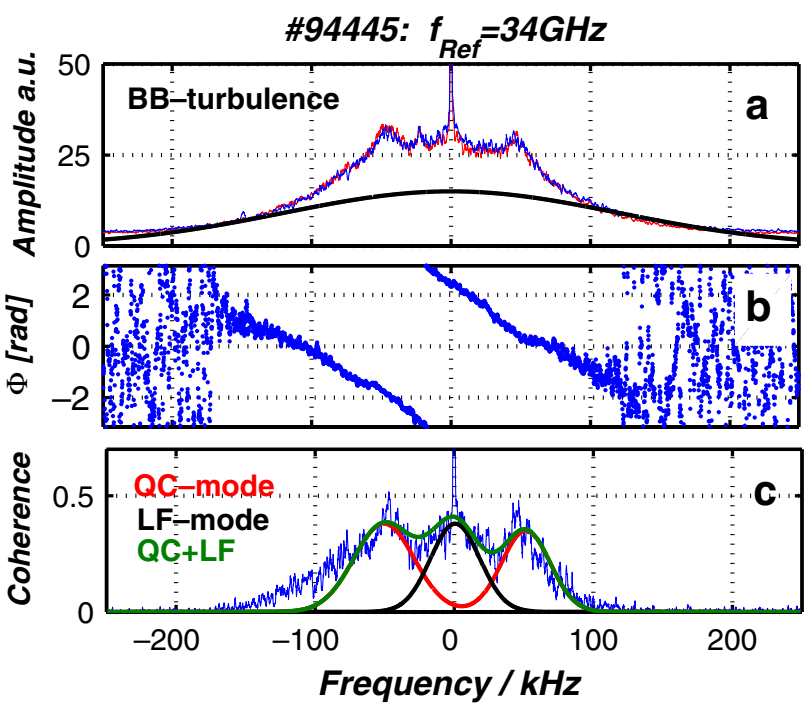

Figure 4. (a) Amplitude-, (b) cross phase and (c) coherence spectrum obtained from two antennas. The coherence spectrum shows the presence of LF- and QC-modes, which are coloured coded. The BB-turbulence is only sketched in the amplitude spectrum. The cross phase can be estimated from QC- and LF-modes only. LF- and QC-modes have the same slope, showing that they rotate with the same frequency.

shows a typical example of the spectra obtained from two arbitrarily chosen antennas from the midplane array. The upper spectrum (see figure 4(a)) shows the amplitude of the reflected signal of two antennas. Besides the BB turbulence (sketched by the solid line) two other fluctuation modes can be distinguished, the QC [5] and the LF [5] modes. The QC-mode is recognized by the two broad peaks at $\pm 50 \mathrm{kHz}$ in figure $4(a)$. The long-living, high $m$-number QC mode is detected mostly in the central and gradient region of the plasma. It is represented by a peak with $(f / \Delta f)_{\mathrm{QC}} \approx 1-3$ (see figure $4(a)$ ), where $f$ is the centre frequency of the QC-mode and $\Delta f$ is the width of the mode. The LF mode occupies the LF region in the spectra. These long-scale fluctuations have a Gaussianlike spectrum centred at zero frequency (see figure 4(a)). It is always observed at the periphery and in plasmas with low confinement (L-mode) in the gradient region as well.

The decorrelation time of both modes is large enough for delay measurements and can therefore be used for turbulence propagation analysis. Past experiments on TEXTOR show that the turbulence rotation for the centre and gradient regions of the plasma can be well calculated from the QC-mode propagation. For plasmas with developed turbulence as for the additional heated plasmas by neutral beam injection and for the plasma periphery, where the QC-mode is hardly visible, the turbulence rotation is calculated from LF-mode propagation.

The propagation of these modes can be found from the cross-phase $\Phi(f)$ spectrum (see figure $4(b)$ ) as long as $\mathrm{d} \Phi / \mathrm{d} f$ is inversely proportional to the angular velocity of the turbulence $\Omega_{\text {turb }}$ by:

$$
\Omega_{\mathrm{turb}}=\frac{2 \pi \cdot \Delta \Theta}{\mathrm{d} \Phi / \mathrm{d} f},
$$

where $\Delta \Theta$ is the poloidal angle between reflecting spots. If the different types of fluctuations which compose the resulting 
spectrum propagate with approximately the same velocity the slope of the cross-phase should be constant over the whole frequency region (see figure $4(b)$ ). The different velocities of propagation of separate modes can lead to velocity dispersion over the spectrum. In the case of large overlapping of modes in the spectrum, the propagation analysis becomes difficult.

The coherence $(\gamma)$ spectrum (see figure $4(c)$ ) shows the degree of correlation for the Fourier components of two signals. A sufficient value of coherence, together with a small scattering in $\Phi(f)$, guarantees a confident measurement of the turbulence propagation.

The time delay analysis makes it possible to deduce the propagation according to

$$
\Omega_{\mathrm{turb}}=\frac{\Delta \Theta}{\Delta t},
$$

where $\Delta t$ is the time delay between two signals and is derived from the shift with respect to zero of the maximum of the crosscorrelation function (CCF). Assuming a strong elongation of the density perturbations along the magnetic field lines, the calculated $\Omega_{\text {turb }}$ is, in principle, the component perpendicular to the magnetic field. Therefore $\Omega_{\text {turb }}$ is determined by the poloidal and toroidal plasma rotations. To choose the frequency range with the highest coherence, the smallest scattering in $\Phi(f)$ and an absence of dispersion, an appropriate bandpass-filtering of the signals is used. To keep the accuracy of the $\Delta t$ measurements high, a series of successive CCFs must be averaged. The length of the analysed series depends on the turbulence properties. A sample averaging of 20 and sample length of 1024 is used in the presented analysis. The accuracy of the angular velocity estimation is further increased by taking into account all poloidally distinct combinations of one antenna array.

The linear turbulence velocity $\left(v_{\text {turb }}\right)$, the poloidal wavelength $\left(\lambda_{\perp}\right)$ and the wave number $\left(k_{\perp}\right)$ are calculated from the knowledge of the cutoff radius $r_{c}$. The cutoff radius is deduced, using the $\mathrm{HCN}$-interferometer at TEXTOR, with an accuracy of $\Delta r_{c}= \pm 1 \mathrm{~cm}$.

For coherent structures in space, as in the QC mode, it is possible to determine the wave-length merely from its centre frequency $f_{\mathrm{QC}}$ in the spectrum by

$$
\lambda_{\perp}^{\mathrm{QC}}=\frac{\Omega_{\mathrm{turb}} \cdot r_{c}}{f_{\mathrm{QC}}}, \quad k_{\perp}=\frac{2 \pi}{\lambda_{\perp}} .
$$

Normally in the periphery of the plasma the QC mode is not detectable and the spectrum consists of the LF mode and BB turbulence as mentioned above. Both modes exhibit a smooth Gaussian-like spectra with an approximate full width at half maximum (FWHM) $\Delta f=20-50 \mathrm{kHz}$ for LF and $\Delta f \geqslant 300 \mathrm{kHz}$ for $\mathrm{BB}$ turbulence. If the toroidal rotation is negligible it is possible to estimate the turbulence wavelength from the auto-correlation function (ACF). The perpendicular wavelength is determined by

$$
\lambda_{\perp} \cong v_{\mathrm{turb}} \cdot \sigma_{\mathrm{ACF}}=\Omega_{\mathrm{turb}} \cdot r_{c} \cdot \sigma_{\mathrm{ACF}},
$$

where $\sigma_{\mathrm{ACF}}$ denotes the FWHM of the ACF in seconds. However in the case of large toroidal rotation the above method is not correct because $\Omega_{\text {turb }}$ is a perpendicular projection with respect to the main magnetic field. In this case, the measurements from a sequence of poloidallyistributed antennas can provide information on the turbulence wavelength provided that the minimum distance between the probing areas is at least half of the wavelength. Due to the antenna geometry at TEXTOR, the distance between reflecting spots increases with $r_{c}$. For typical cutoff radii $0.20 \leqslant r_{c} \leqslant$ $0.42 \mathrm{~m}$, the minimum poloidal distance varies from 0.005 to $0.01 \mathrm{~m}$. This means that perturbations with $\lambda_{\perp} \geqslant 0.01 \mathrm{~m}$ in the gradient region and with $\lambda_{\perp} \geqslant 0.02 \mathrm{~m}$ for the periphery fulfils the requirement. In practice, the $\mathrm{CCF}$ at zero lag $\mathrm{CCF}^{0}\left(x_{i}\right)$ is calculated for every $x_{i}=r_{c} \cdot \Delta \Theta_{i}$. The resulting data can be approximated by a Gaussian shape. The FWHM of this curve is a measure of the average perpendicular wavelength of fluctuations in the frequency range under consideration.

The lifetime of density fluctuations can be considered as a measure of the growth rate of instabilities. The developed instabilities reveal a nonlinear behaviour with their amplitude mostly saturated and restricted. On the contrary small fluctuations with a small linear growth rate, in the absence of nonlinear effects, have a longer evolution time. The finite lifetime of fluctuations can be deduced from the crosscorrelation coefficients $(\gamma)$ for several spatially distributed antennas and known turbulence velocity. A larger poloidal distance between reflecting spots results in an increased delay and a decreased correlation between the signals, assuming a constant turbulence velocity. The distribution of $\gamma$ at different poloidal distances $\left(\Delta \Theta_{i}\right)$ is described by a Gaussian line shape. From the FWHM of this distribution, $l_{c}$, which is an upper bound of the average lifetime or decorrelation time (with errors of $10-30 \%$ ) of the fluctuations, is evaluated:

$$
\tau_{\mathrm{dc}}=\frac{l_{c}}{v_{\mathrm{turb}}}
$$

It should be kept in mind that within the framework of Fourier analysis it is impossible to distinguish between LFmode and BB-turbulence since BB-turbulence occupies the whole frequency range and can influence the results obtained either for LF or QC modes. Since QC- and LF-modes occupy different frequency regions it is possible to discriminate between them by applying appropriate filters. However, for the propagation analysis this is not crucial because BBturbulence has the same velocity [5]. For the decorrelation time analysis described above the BB-turbulence has a much smaller decorrelation time $(\leqslant 1 \mu \mathrm{s})$ compared with QC- and LF-modes [22] and acts as uncorrelated noise.

The density fluctuation level [7] can be estimated from a single antenna according to

$$
\frac{\left\langle|\delta n|^{2}\right\rangle^{1 / 2}}{n_{c}} \approx\langle\tilde{\Phi}\rangle \cdot \frac{\lambda_{0}}{4 \pi \cdot 1.5 \cdot 2^{3 / 4} \sqrt{L_{n} \lambda_{\perp}}},
$$

where $\langle\tilde{\Phi}\rangle$ is the phase fluctuation level in radians, $\lambda_{0}$ is the vacuum wavelength of the probing microwave and $L_{n}$ is the density scale length. For the estimation of $\langle\tilde{\Phi}\rangle$ the mean for four antennas is calculated. The accuracy of such an evaluation is satisfactory for the central and gradient regions of the plasma but gives large errors at the periphery and in L-mode plasmas, when the turbulence is developed to such degree that the interference effects in the amplitude of the signal and phase jumps lead to a random phase distribution between 0 to $2 \pi$. The further growth of the turbulence does not yield a further growth of $\langle\tilde{\Phi}\rangle$. In this case $\langle\tilde{\Phi}\rangle$ is therefore saturated. 


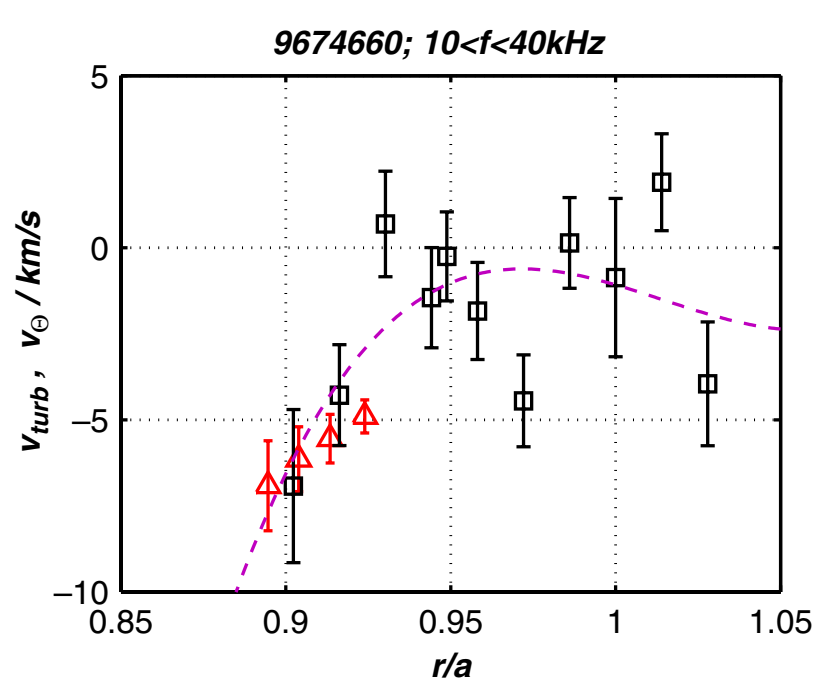

Figure 5. Comparison between $v_{\Theta}$ from Doppler-shifted carbon lines $(\square)$ and $v_{\text {turb }}$ from reflectometry $(\Delta)$. The dashed line is obtained from a 3rd order polynomial approximation to the carbon data and visualizes the trend of the data. Good agreement at $r / a \approx 0.9$ is found

\subsection{Comparison between turbulence and plasma rotation}

For the analysis of transport properties the knowledge of the velocity shear is of interest. In principle the toroidal plasma velocity is measured by CXRS. However at TEXTOR these data are available for $r / a \leqslant 0.6$ only. Since the changes in the velocity shear due to the DED are expected at the plasma edge, only reflectometry measurements can close the gap between CXRS measurements, using Doppler shifted carbon ions, in the core and at the plasma boundary [23]. For the latter method it is assumed that the poloidal velocity of carbon ions is the same as the velocity of the background plasma.

In general the turbulence velocity from reflectometry $v_{\text {turb }}$ is described by

$$
v_{\text {turb }}=v_{\perp}+v_{\text {phase }}, \quad v_{\perp}=v_{\Phi} \frac{B_{\Theta}}{B}-v_{\Theta} \frac{B_{\Phi}}{B},
$$

where $v_{\text {phase }}$ is an additional velocity of the fluctuations in the plasma frame, $B_{\Theta}$ the poloidal and $B_{\Phi}$ the toroidal magnetic field. The perpendicular (to the main magnetic field) plasma velocity $v_{\perp}$ is the superposition of the toroidal plasma rotation $v_{\Phi}$ and the poloidal plasma rotation $v_{\Theta}$. Furthermore $B_{\Theta}$ is obtained from a standard current profile [24] of the form $j=j_{0} \cdot\left(1-(r / a)^{2}\right)^{3}$. In the plasma core the measurement of $v_{\Theta}$ is not available and it is calculated from neoclassical theory [25].

$$
v_{\Theta}=k \cdot \frac{c}{e \cdot B_{t}} \cdot \nabla T_{i}
$$

From equation (8) it is seen that $v_{\Theta}$ depends on the temperature gradient and $k$ is a numerical coefficient depending on the collisionality of the plasma.

At the plasma boundary $v_{\text {turb }}$ is obtained from the LF-mode and can directly be compared with $v_{\Theta}$. At $r / a \approx 0.9, v_{\Phi}$ can be neglected for a first approximation, yielding $v_{\perp} \approx v_{\Theta}$. As can be seen in figure 5 an agreement within the error bars of $v_{\Theta}-$ the velocity is estimated from Doppler shifted carbon linesand $v_{\text {turb }}$ has been obtained for a small overlapping region. The dashed line in figure 5 is obtained from a 3rd order polynomial approximation of the data from Doppler shifted carbon ions and describes the trend in the data. This good consistency is an indication that the LF-mode has nil or negligible phase velocity with respect to the poloidal plasma velocity.

For a comparison of $v_{\perp}$ with $v_{\text {turb }}$ in the gradient region, estimated from the QC-mode, $\bar{n}_{e}$ must be small to have $v_{\Phi}$ and $v_{\text {turb }}$ measurements at the same radius. Even if the radial overlap of reflectometry and CXRS is small a comparison is possible. However to avoid inaccuracies in the ion temperature measurements by CXRS at the boundary of the observation range, the electron temperature gradient $\left(\nabla T_{e}\right)$ is used instead in the calculation of $v_{\Theta}$ in equation (8). For the data under investigation this is justified because approximately equal ion and electron temperatures are measured in the plasma centre. The investigated discharges are extreme cases of induced toroidal rotation in the counter-current and cocurrent directions. For the counter-current direction the central toroidal rotation is $v_{\Phi}=-155 \mathrm{~km} \mathrm{~s}^{-1}$, whereas $v_{\Phi}=105 \mathrm{~km} \mathrm{~s}^{-1}$ is measured for the co-current direction. In figure $6(a)$ the different components of equation (7) for $v_{\perp}$ at $r / a \approx 0.5$ are shown for a discharge predominately heated by counter injection $\left(P_{\mathrm{NBI}}=1.3 \mathrm{MW}\right)$ with a small amount of co-injection $\left(P_{\mathrm{NBI}}=0.3 \mathrm{MW}\right)$ for CXRS measurements. A good agreement between the calculated $v_{\perp}$ and the turbulence velocity is found for the flat-top phase $(1.2 \leqslant t \leqslant 2.4 \mathrm{~s})$, where $r_{c}$ is kept constant. The same good agreement is found for a discharge with pure co injection $P_{\mathrm{NBI}}=0.8 \mathrm{MW}$ (see figure $6(b)$ ). The scatter on the $v_{\text {turb }}$-curve arises from the decreased amplitude of the QC-mode in this particular discharge. Both results confirm that, within the accuracy of the measurement, $v_{\text {turb }}$, deduced from the QC-mode, has none or negligible phase velocity with respect to the plasma rotation. If $v_{\text {phase }} \neq 0$ is assumed and if $v_{\text {phase }}$ depends on local quantities for instance on $\nabla T_{e}$ or $\nabla n_{e}$, the contribution to $v_{\text {turb }}$ is an additional offset, always in the same direction either in co- or counter-current direction. An assumed additional offset ( $\left.v_{\text {phase }}\right)$ yields then, in the case of a change in the plasma rotation direction from co- to counter-current direction, either $v_{\text {turb }} \leqslant v_{\perp}$ or $v_{\text {turb }} \geqslant v_{\perp}$. However this is not confirmed by the measurements.

The results from the gradient region and the plasma boundary support the idea that the two types of fluctuations propagate with the plasma velocity and have nil or negligible additional phase velocity. From this result the estimation of the radial electric field $E_{r}$ is possible according to

$$
v_{E \times B}=v_{\text {turb }}-v_{\text {dia }}, \quad E_{r}=\left(v_{\text {turb }}-v_{\text {dia }}\right) \cdot B,
$$

where $v_{E \times B}$ is the poloidal drift velocity due to the radial electric field. Here $v_{\perp}$ is replaced by $v_{\text {turb }}$ and $v_{\text {dia }}$ is the diamagnetic velocity, which can be deduced from $T_{e}$ measurements obtained using the ECE diagnostic and the density scale length $L_{n}$.

\subsection{Relation to turbulent transport}

A question of great interest is the effect of the turbulence on the transport properties. One of the key parameters for radial turbulent transport is the radial wavelength $\lambda_{r}$. Therefore measurements with two or more different frequencies should 

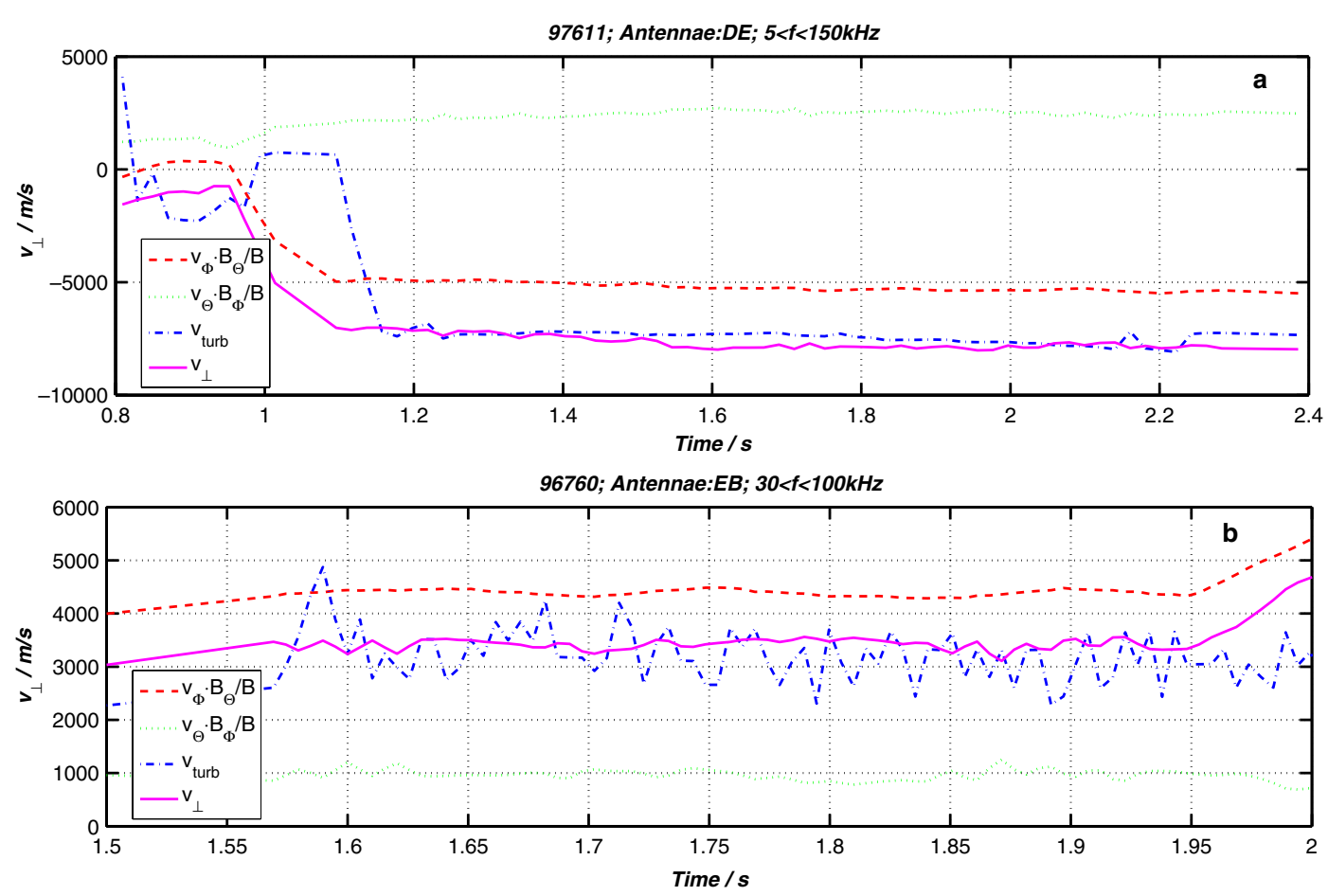

Figure 6. Comparison of $v_{\perp}$ with $v_{\text {turb }}$. The poloidal and toroidal components of $v_{\perp}$ are also shown. (a) rotation in the counter-current direction $\left(P_{\text {counter }}=1.3 \mathrm{MW}\right.$ and $\left.P_{\mathrm{co}}=0.3 \mathrm{MW}\right)$ and $(b)$ rotation in the co-current direction $\left(P_{\mathrm{co}}=0.8 \mathrm{MW}\right)$. The good agreement between $v_{\perp}$ and $v_{\text {turb }}$ suggests a negligible phase velocity of the turbulence.

be performed simultaneously, which are not available at TEXTOR at this time. However experiments with beam emission spectroscopy at TFTR [4] and T-10 [5] have shown that $\lambda_{\Theta} \approx 2 \cdot \lambda_{r}$. Furthermore, the underlying transport model is also of importance. A simple model is to describe the transport by a random walk process [26]. The diffusion coefficient $\left(D_{\mathrm{RW}}\right)$ is related to the turbulence properties by

$$
D_{\mathrm{RW}}=\frac{\lambda_{r}^{2}}{\tau_{\mathrm{dc}}},
$$

where $\lambda_{r}$ and $\tau_{\mathrm{dc}}$ can be determined from reflectometry measurements.

\section{Experimental observations with DED}

\subsection{DED in the $3 / 1$ configuration with Co-injection (Scenario 1)}

For Scenario 1, the observation range of the reflectometry is limited to $0.75 \leqslant r / a \leqslant 0.95$. Note that the measurements of the reflectometry are well outside the $q=2$ island (see section 3). The observations are divided into three periods; (i) plasma without DED, (ii) plasma with DED and (iii) plasma with DED and locked mode. The turbulence rotation was investigated in all three cases. Without DED the turbulence velocity is determined from both the QC-mode and the LFmode. Both QC- and LF-modes are visible with a small amount of additional heating. The turbulence propagation is found to be in the electron diamagnetic drift (edd) direction. At $r / a=0.9$ the amplitude of the QC-mode decreases with the onset of the DED and vanishes completely when the locked mode is generated in the plasma. In this case the turbulence

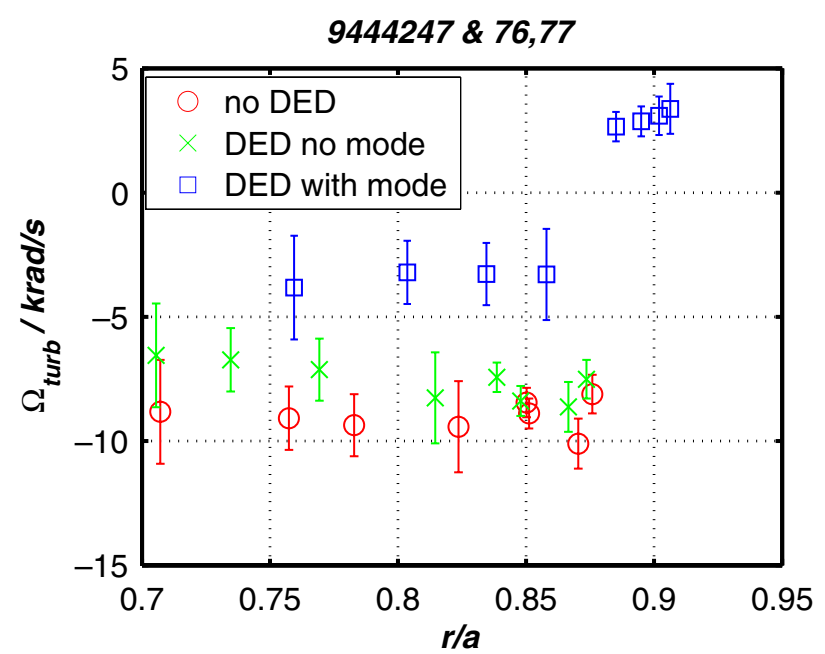

Figure 7. $\Omega_{\text {turb }}$ from LF-mode for three different cases: without $\operatorname{DED}(\mathrm{O})$; with DED below the threshold for the $2 / 1$ island $(x)$ and with DED and the $2 / 1$ island $(\square)$. A clear change of $\Omega_{\text {turb }}$ to the ion diamagnetic drift direction is observed with the DED. For

$r / a \geqslant 0.9, \Omega_{\text {turb }}$ changes from the electron- to the ion-diamagnetic drift direction.

rotation is estimated from the LF-mode only. In figure 7 the results of $\Omega_{\text {turb }}$ versus $r / a$ are summarized. In the investigated radial region the turbulence rotation without DED is constant ( $\square$ in figure 7). During the linear increase of the DED current and before the onset of the locked $2 / 1$ island a decrease of $\approx 20 \%$ in $\Omega_{\text {turb }}$ for $0.75 \leqslant r / a \leqslant 0.85$ is observed. This is interpreted as a braking of the turbulence rotation by the DED. The braking of the plasma rotation by the DED and the flat toroidal rotation profiles results in a locked $2 / 1$ mode. With the 


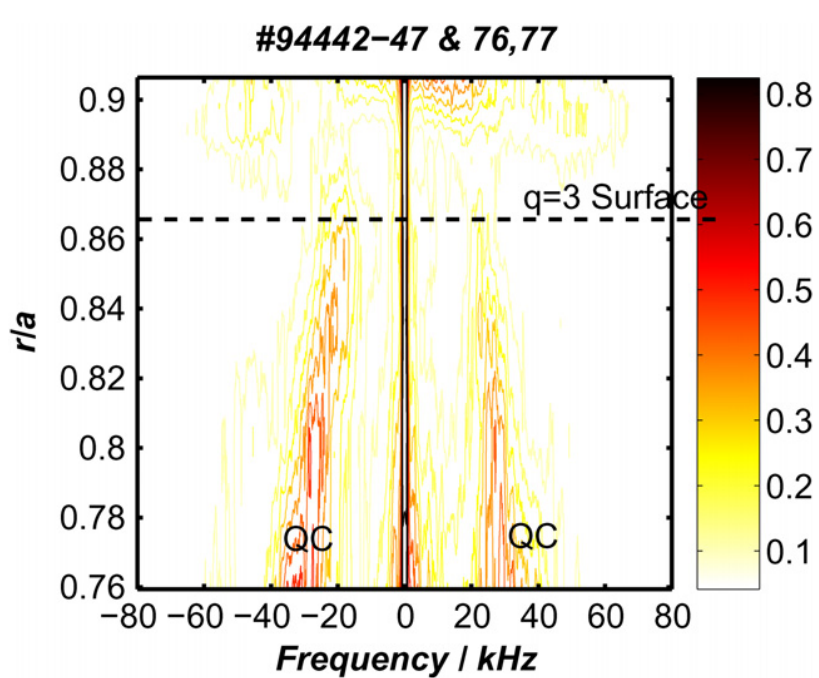

Figure 8. The evolution of the coherency shows a decrease in the QC centre frequency as well as a reduction in the coherence amplitude when the DED has generated the locked mode. The time window for the analysis is $2.5 \leqslant t \leqslant 3.0 \mathrm{~s}$. At $q=3$, the QC-mode vanishes. LF-turbulence is observed outside the $q=3$ only.

onset of the locked mode the perpendicular turbulence rotation decreases rapidly and around the $q=3$ surface the rotation direction changes from electron to ion diamagnetic drift (idd) direction (see figure 7). In this case $\left|\Omega_{\text {turb }}\right|$ is the same inside and outside the $q=3$ surface. The contour representation of the coherence (figure 8) shows (i) a damping of the QC mode and (ii) a radial decrease of its central frequency. This reflects the slow down of the turbulence rotation. At the $q=3$ surface and its vicinity, the QC-mode and the LF-mode have vanished completely. This suppression can be explained by the large variation in the rotation at $r / a=0.9$, as shown in figure 7 .

Due to the suppression of the QC-mode in the DED phase, the estimation of the perpendicular wavelength averaged in time $\left\langle\lambda_{\perp}\right\rangle$ is performed from the LF-mode for all three stages. The frequency range chosen for this estimations is $1 \leqslant f \leqslant$ $30 \mathrm{kHz}$. Without the DED we observe a slight increase of $\left\langle\lambda_{\perp}\right\rangle$ approaching $\left\langle\lambda_{\perp}\right\rangle=0.055 \mathrm{~m}$ at the plasma edge (figure $9(a)$ ), corresponding to a poloidal wave number $m \approx 60$. Already for $I_{\mathrm{DED}} \leqslant 0.75 \mathrm{kA}$ a significant decrease of $\left\langle\lambda_{\perp}\right\rangle$ by $25 \%$ (see $\times$ in figure $9(a)$ ) as well as an increase in the poloidal wave number of $\Delta m \approx 10$ is observed. When the $2 / 1$ mode locks, the turbulence wavelength drops to $\left\langle\lambda_{\perp}\right\rangle \approx 0.02 \mathrm{~m}$ (see $\square$ in figure $9(a)$ ) and is constant over the whole investigated radial region.

The turbulence lifetime $\left(\tau_{\mathrm{dc}}\right)$ is shown in figure $9(b)$. With $I_{\text {DED }} \leqslant 0.75 \mathrm{kA}, \tau_{\mathrm{dc}}=13 \pm 1 \mu \mathrm{s}$ was estimated and is independent of the radial position. For the time interval with the locked $2 / 1$ mode the estimation of $\tau_{\mathrm{dc}}$ becomes difficult due to the decrease in $\Omega_{\text {turb }}$. At the most inboard radius, $\tau_{\mathrm{dc}}=22 \mu \mathrm{s}$ is obtained, an increase by a factor $\approx 2$ compared with the non-DED plasma. At $r / a \approx 0.9 \tau_{\mathrm{dc}} \approx 12 \mu$ s was deduced, which is similar to the values without DED.

The estimation of the density fluctuation level, according to equation (6), is based on the phase fluctuation $\langle\tilde{\Phi}\rangle, \lambda_{\perp}$ and $L_{n}$. Since the reflectometry measurements are performed close to the X-point of the $2 / 1$ locked mode, $L_{n}$ is not expected to change much. The observations indeed show a decrease

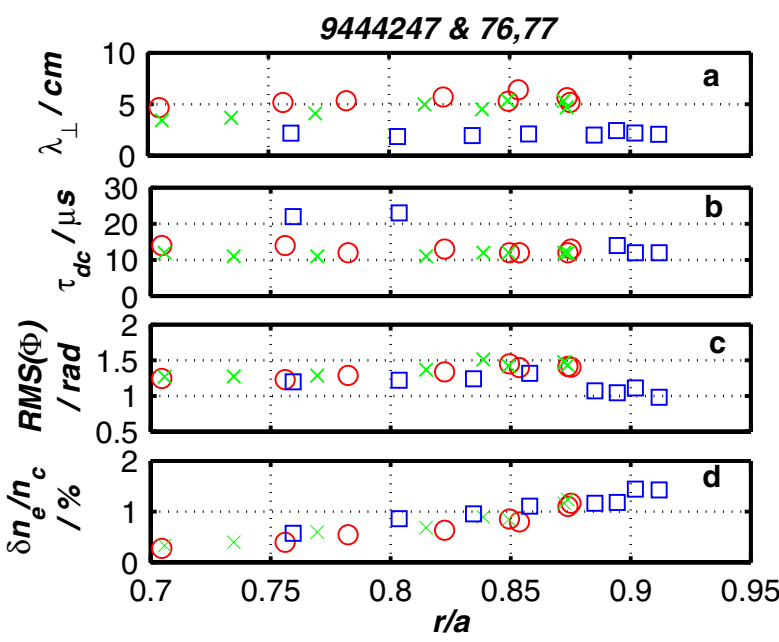

Figure 9. (a) $\left\langle\lambda_{\perp}\right\rangle,(b) \tau_{\mathrm{dc}},(c)$ phase fluctuations and $(d) \delta n / n_{c}$ for different periods. Without the DED $(O)$, with the DED $(x)$ and with the DED and the 2/1 locked mode $(\square)$. A decrease in $\left\langle\lambda_{\perp}\right\rangle$ by $50 \%$ is observed. The reduction in the phase fluctuations is compensated by the decrease in $\left\langle\lambda_{\perp}\right\rangle$ and therefore no change in $\delta n / n_{c}$ is found.

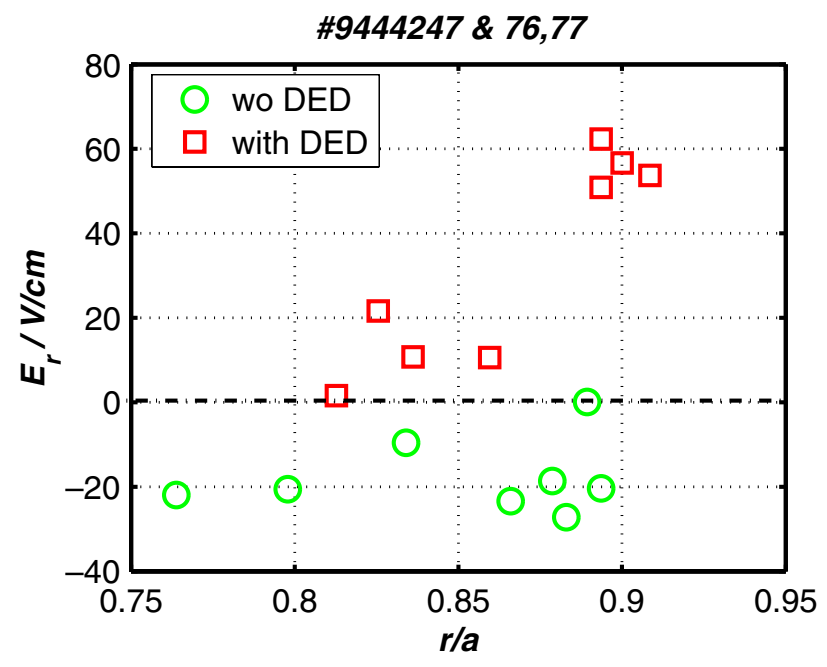

Figure 10. Radial electric field estimated using equation (9). $\bigcirc$ denotes plasmas without DED. $\square$ denote plasmas with DED in the $3 / 1$ configuration. With the DED, $E_{r}$ becomes positive and shifts the inversion point more deeply into the plasma to $r / a \approx 0.8$.

in $\langle\tilde{\Phi}\rangle$ when the mode locks. However it is compensated by the decrease in $\lambda_{\perp}$ (figure $9(a)$ ). As a consequence the deduced density fluctuation level $\delta n / n_{c}$ is not changed after mode locking. For all three periods $\delta n / n_{c}$ increases towards the plasma edge, indicating an enhanced transport at the plasma edge due to a resistive interchange mode.

From equation (9) the radial electric field $\left(E_{r}\right)$ is estimated in DED and non-DED plasmas. The diamagnetic velocity $v_{\text {dia }}$ is calculated from the electron density and temperature which are measured using the HCN interferometer and ECE diagnostic, respectively. For plasmas without DED, a negative $E_{r}=-20 \mathrm{~V} \mathrm{~cm}^{-1}$ is found for $0.75 \leqslant r / a \leqslant 0.9$. However, with a DED current above the threshold for the onset of the locked 2/1-mode, $E_{r}$ becomes positive and increases with radius (see figure 10). With DED a change in the sign of $E_{r}$ at $r / a \approx 0.9$ is most probably an indication of an inward 

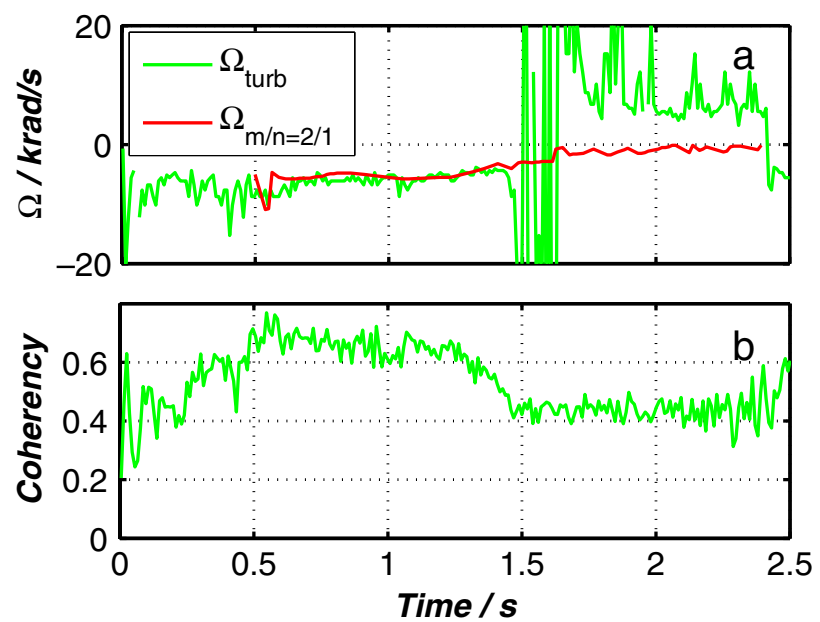

Figure 11. (a) Comparison between $\Omega_{\text {turb }}$ and $\Omega_{2 / 1}$ from ECE measurements. As long as the island width is small, $\Omega_{\text {turb }}$ and $\Omega_{2 / 1}$ are in good agreement. When the $2 / 1$ island slows down, the rotation decouples and $\Omega_{\text {turb }}$ changes to the idd direction. (b) Even for the locked mode the coherency is large enough to have reliable $\Omega_{\text {turb }}$ measurements.

movement of the inversion point. From the change in the turbulence spectrum of the laser scattering diagnostic [13] at Tore Supra (during ergodic divertor operation) an inward movement of the inversion point was also supposed.

The question at this point is whether the measured effects can be attributed to the DED itself or to the locked mode. Therefore, a discharge with the same plasma parameters as above but without the DED and auxiliary heating is analysed and in this case, a large, locked $2 / 1$ island develops in the plasma. The reflection layer is again located outside of the island at $r / a \approx 0.9$. The turbulence rotation is estimated in the range $10 \leqslant f \leqslant 30 \mathrm{kHz}$ which is well above the natural frequency of the $2 / 1$ mode. The rotation of the $2 / 1$ mode is calculated from a Fourier spectrum of an ECE-channel close to the island on the LFS. As long as the mode is stable, $\Omega_{\text {turb }}$ coincides well with the mode rotation $\left(\Omega_{2 / 1}\right)$ (figure 11(a)). As soon as the mode starts to grow in amplitude, its rotation brakes and then the turbulence and mode rotation start to decouple. A critical parameter for the onset of the decoupling seems to be the island width, which is $w \approx 0.06 \mathrm{~m}$ when the decoupling starts. This is about the same value as observed for the locked mode generated by the DED. When the $2 / 1$ mode starts to lock, the LF-mode changes the rotation direction to the idd direction. Besides a short period where the turbulence rotation changes sign, the coherency is high enough to allow the above conclusions (see figure 11(b)). The observation is an indication that the change in the turbulence rotation is mainly caused by the large island and not by the DED itself.

\subsection{DED in the $3 / 1$ configuration with counter-injection (Scenario 2)}

As mentioned in section 5.1, the DED brakes the plasma rotation at the plasma edge. However, with co-current NBI, a large 2/1-mode is generated due to the flat toroidal rotation profile. With counter-current NBI the rotation profile stays steep. The favourable role counter injection in the generation of internal transport barriers has been reported earlier by
\#97597-606 Coherency with DED

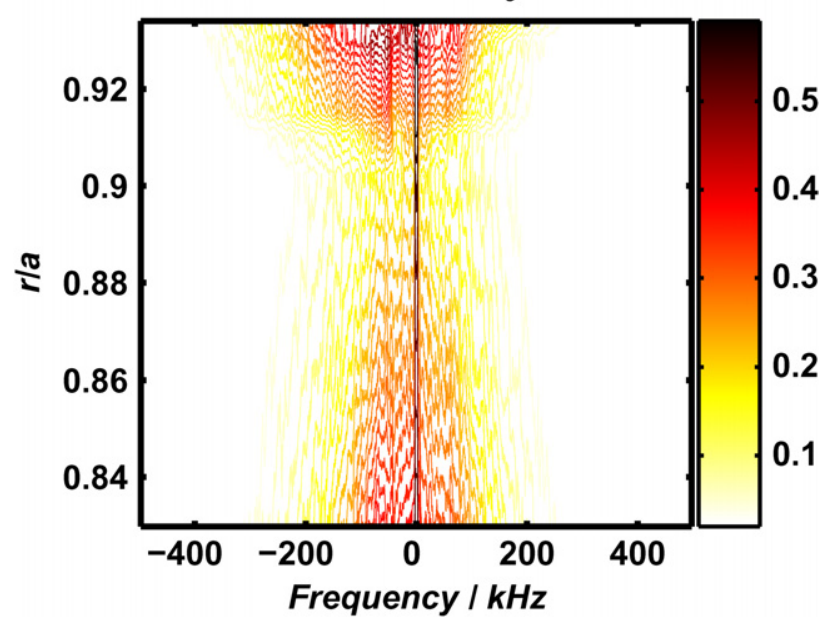

Figure 12. Coherency spectrum versus radius during the DED operation. Due to large NB-injection in the counter-current direction the QC-mode is not visible. A reduction in the coherency at $(r / a \approx 0.91)$ is found. This indicates a decrease in turbulence rotation.

$[27,28]$. Due to the speed up of the poloidal plasma rotation by counter-current injection [7] in the plasma centre and braking at the plasma edge by the DED, a region with increased rotation shear could be generated. This may influence the turbulence transport too. For this scenario reflectometry measurements are performed in the region $0.5 \leqslant r / a \leqslant 0.95$. Note that for the following discussion plasma- and turbulence rotation-are considered to be equal, as discussed in section 4.2.

The spectral analysis shows a broad distribution of the coherence due to fast rotation of the plasma. Without the DED it is centred around $f=0 \mathrm{kHz}$ with a FWHM $\approx 400 \mathrm{kHz}$. The coherence drops by a factor of $\approx 2$ at $r / a=0.9$ and this position coincides with that of the $q=3$ surface. The DED causes a reduction in the width of the coherence distribution to a FWHM $\approx 200 \mathrm{kHz}$ and is even more reduced at $r / a=0.9$ (figure 12). This is an indication of a drop in the turbulence rotation.

The measurements of $\Omega_{\text {turb }}$ for $5 \mathrm{kHz} \leqslant f \leqslant 150 \mathrm{kHz}$ with and without DED are shown in figure 13(a). Note that, in the frame of reference, counter rotation yields negative values (edd) for $\Omega_{\text {turb }}$. In the case without DED ( $\bigcirc$ symbols), $\left|\Omega_{\text {turb }}\right|$ decreases slightly with radius for $0.7 \leqslant r / a \leqslant 0.85$. For $r / a \leqslant 0.7$ the torque of the counter beam affects the plasma column and the turbulence rotation increases towards the plasma centre. With the DED ( $\star$ symbols), the turbulence rotation is decreased for $0.8 \leqslant r / a \leqslant 0.9$. For $r / a \geqslant 0.9$ the turbulence rotation slightly increases. The maximum of the shear is found at $r / a \approx 0.9$, which is close to the position of the $q=3$ surface $(r / a \approx 0.85)$. For $r / a \leqslant 0.75, \Omega_{\text {turb }}$ is not changed much as can be seen from the coincidence of the $\star$ and $O$ symbols in figure 13(a). This shows that the effect of the DED on the plasma rotation vanishes for $r / a \geqslant 0.75$.

The rotation shear $\left(\omega^{\prime}\right)$ is calculated from the turbulence rotation, assuming that the turbulence rotation equals the plasma rotation (see section 4.2), and is defined by [29]

$$
\omega^{\prime}=\frac{v_{\Theta}\left(r_{2}\right)-v_{\Theta}\left(r_{1}\right)}{r_{2}-r_{1}}, \quad v_{\Theta}=\Omega_{\mathrm{turb}} \cdot r .
$$




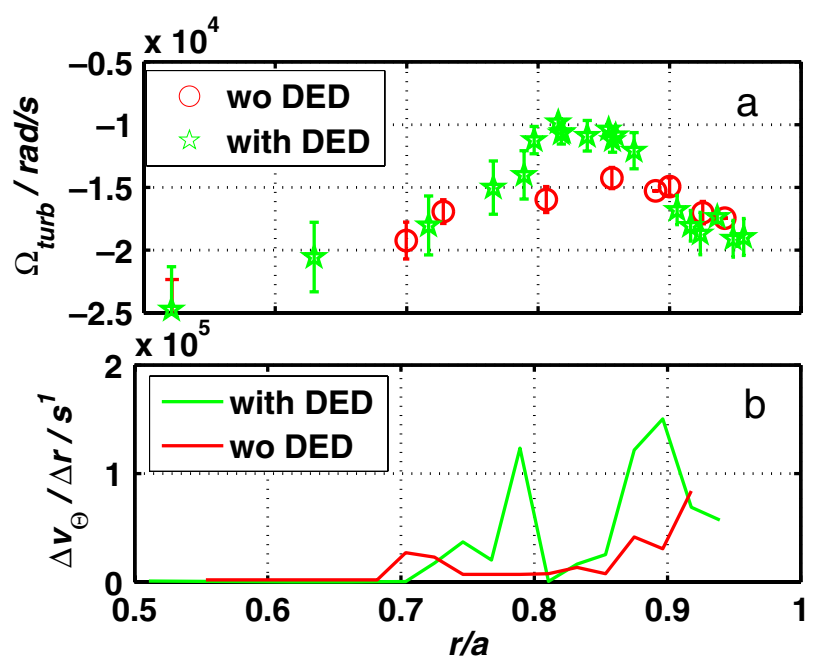

Figure 13. (a) $\Omega_{\text {turb }}$ for counter-current NB-injection. $\bigcirc$ denotes operation without DED and $\star$ with DED. For $0.8 / a \leqslant 0.9$ the rotation is decreased by the DED. (b) As a consequence, a shear in the velocity for the DED case is observed at $r / a=0.8$ and $r / a=0.9$, respectively.

In general, it is found that the rotation shear increases for a region with reduced transport [28]. Without the DED, $\omega^{\prime}$ is flat in the investigated region and only a small increase at the plasma edge is observed (see figure 13(b)). When the DED is applied, $\omega^{\prime}$ increases at $r / a=0.9$ and at $r / a=0.8$ (see figure 13(b)). This indicates the formation of a transport barrier as discussed in [30] and references therein.

Further evidence of the forming of a transport barrier derives from the measurement of the density fluctuation. Since only the electron density is affected, which is seen by the increasing $\bar{n}_{e}$ in figure $2(a)$, the most probable change is expected in the particle channel. This implies a reduction of the density fluctuation level at the same radial position as the increased shear was found. In general an increase in $\delta n / n_{c}$ towards the edge is observed for the cases both with and without DED (see figure 14(a)). For the radial range under investigation an increase from $\delta n / n_{c}=0.5 \%$ to $\delta n / n_{c}=1.8 \%$ is found. When the DED is applied, no change in $\delta n / n_{c}$ is observed for $r / a \leqslant 0.8$, compared with the non-DED case. With the DED applied, $\delta n / n_{c}$ is constant for the range $0.8 \leqslant r / a \leqslant 0.9$. For radii $r / a \geqslant 0.9, \delta n / n_{c}$ in the DED case is lower by $0.2 \%$ when compared with the case without DED.

For the estimation of $\delta n / n_{c}$, the turbulence wavelength had been calculated from the ACF at the plasma edge. In the gradient region, $\left\langle\lambda_{\perp}\right\rangle$ is determined from the CCF at zero lag. The estimation of $\left\langle\lambda_{\perp}\right\rangle$ for $5 \leqslant f \leqslant 150 \mathrm{kHz}$ without DED yields a nearly constant $\left\langle\lambda_{\perp}\right\rangle \approx 0.016 \mathrm{~m}$ with a slight increase towards the plasma edge. When the DED is switched on $\left\langle\lambda_{\perp}\right\rangle$ decreases by $25 \%$ for $0.8 \leqslant r / a \leqslant 0.9$ (see figure $14(b)$ ). As a consequence, the poloidal wave number in this region is increased by the same percentage. At $r / a \approx 0.94$, $\left\langle\lambda_{\perp}\right\rangle=0.027 \mathrm{~m}$ is calculated for the DED plasmas, compared with $\left\langle\lambda_{\perp}\right\rangle=0.022 \mathrm{~m}$ for plasmas without DED. For the radial range $0.50 \leqslant r / a \leqslant 0.75$, no difference in $\left\langle\lambda_{\perp}\right\rangle$ for DED and non-DED plasmas is observed.

The analysis of the turbulence lifetime, estimated from the CCF for different antenna combinations, yields constant

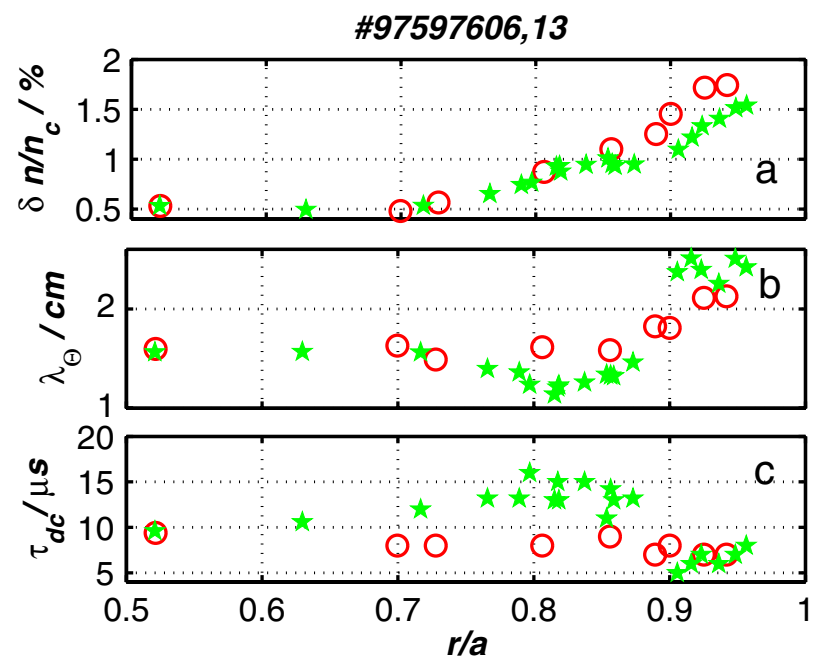

Figure 14. Plasmas with DED are denoted by $\star$ and those without DED by $O$. (a) $\delta n / n_{c}$ is constant for $0.8 \leqslant r / a \leqslant 0.9$. Constant offset in $\delta n / n_{c}$ for $r / a \geqslant 0.9$. (b) $\left\langle\lambda_{\perp}\right\rangle$ shows a reduction for $0.8 \leqslant r / a \leqslant 0.9$ and $(c) \tau_{\mathrm{dc}}$ is increased for $0.8 \leqslant r / a \leqslant 0.9$. All three quantities point in the direction of reduced transport with DED operation. For $r / a \leqslant 0.75$, no difference between DED and non-DED is observed.

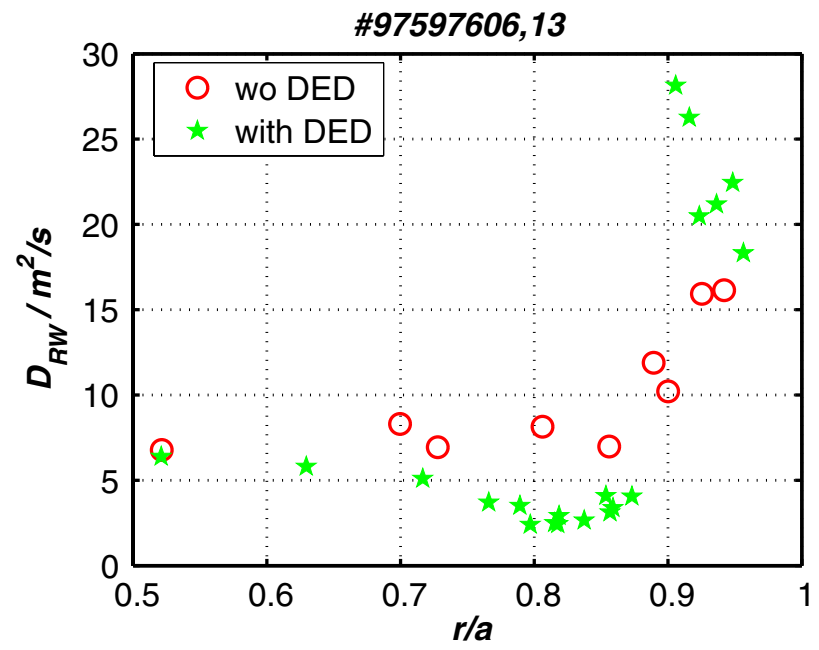

Figure 15. Diffusion coefficients determined from the turbulence properties and compared for DED and non-DED plasmas. Applying the DED reduces $D_{\mathrm{RW}}$ in the region $0.75 \leqslant r / a \leqslant 0.85$. In the laminar zone, $D_{\mathrm{RW}}$ increases again.

$\tau_{\mathrm{dc}} \approx 8 \mu$ s for the investigated radial range in the non-DED plasmas. An increase in the turbulence lifetime to $\tau_{\mathrm{dc}}=13 \mu \mathrm{s}$ was found in the DED case for $0.75 \leqslant r / a \leqslant 0.9$ (see figure $14(c)$ ). Comparing $1 / \tau_{\mathrm{dc}}$ with the shearing rate due to the DED at $r / a=0.9, \omega^{\prime}$ exceeds $1 / \tau_{\mathrm{dc}}$. This means that the conditions for turbulence suppression is fulfilled.

Finally, the electron density scale length $\left(L_{n}\right)$ is analysed. However, due to the large spacing of the $\mathrm{HCN}$ interferometer channels only a crude estimation is possible. Taking the splined profiles, a systematic decrease of $L_{n}$ at $r / a \approx 0.95$ could be deduced. No change in $L_{n}$ was found for $r / a \leqslant 0.90$.

With the knowledge of $\left\langle\lambda_{\perp}\right\rangle$ and $\tau_{\mathrm{dc}}$ the transport coefficient $D_{\mathrm{RW}}$ (equation (10)), for a random walk model, is calculated. In figure $15, D_{\mathrm{RW}}$ for DED plasmas is decreased by 


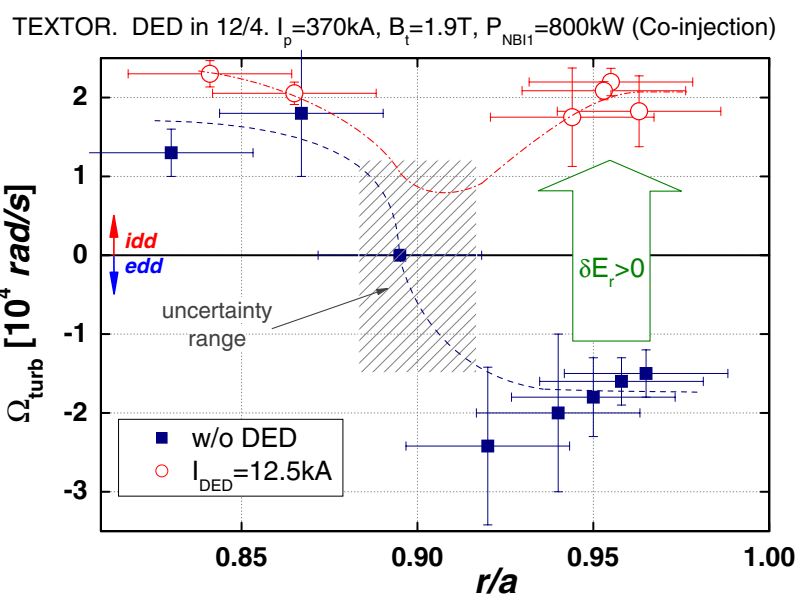

Figure 16. Angular velocity of the turbulence rotation $\Omega_{\text {turb }}$ for DED (in 12/4) and non-DED operation. Dashed and dashed-dotted lines are the approximate trends of data. The region where delay measurements are impossible due to the slowdown of rotation is shown by the hatched rectangle. The DED operation, for $r / a \geqslant 0.94$, changes the poloidal rotation direction in the idd direction-similarly by the SOL plasma. No effect of the DED is found for $r / a \leqslant 0.87$-the idd rotation here is caused by sufficient NBI co-injection. The interpretation of $\Omega_{\text {turb }}(r)$ in

$0.88 \leqslant r / a \leqslant 0.93$ is possible only in the non-DED case, where the rotation is reversed. In the DED a reduced rotation in this range is assumed.

a factor of $2-3$ for the region $0.8 \leqslant r / a \leqslant 0.9$. For $r / a \geqslant 0.9$, $D_{\mathrm{RW}}$ is enhanced most probably due to the generation of a stochastic layer by the DED at the plasma edge. No effect of the DED is found for $r / a \leqslant 0.75$.

All described observations support the existence of a region with reduced transport for $0.8 \leqslant r / a \leqslant 0.9$. Moreover, the experiment confirms that sheared $E \times B$ flows [31] cause a suppression of the long wavelength (small $k_{\Theta}$ ) turbulence. This is the range where the reflectometry system at TEXTOR is most sensitive. Furthermore, it is demonstrated that the DED in the $3 / 1$ configuration indeed has the capability to influence the $E \times B$ flow at the plasma edge, leading to the generation of an internal transport barrier. The width of the barrier can be estimated from the radial distance between the two positions of increased velocity shear, as seen in figure $13(b)$. However, from the performed experiments a relationship between the $q=3$ surface and the generation of the transport barrier is not yet determined. The major change, compared with the results in section 5.1, is the strong tangential neutral beam injection in the counter-current direction which prevents the generation of a locked $m / n=2 / 1$ mode.

\subsection{DED in the $12 / 4$ configuration (Scenario 3 )}

For this configuration the rotation turbulence profiles are shown in figure 16. Independent of the DED operation, for $r / a<0.88, \Omega_{\text {turb }}$ is in the ion diamagnetic drift (idd) direction due to the large toroidal momentum of NBI in the co-current direction. In plasmas without DED the influence of NBI momentum becomes weaker further in the plasma periphery $(r / a \approx 0.90)$ and $\Omega_{\text {turb }}$ changes to the electron diamagnetic drift (edd) direction. This inversion in $\Omega_{\text {turb }}$ is expected at $r / a \approx 0.89$ (see figure 16 ) in plasmas without DED. For DED
TEXTOR. DED in 12/4. $\mathrm{I}_{\mathrm{p}}=370 \mathrm{kA}, \mathrm{B}_{\mathrm{t}}=1.9 \mathrm{~T}, \mathrm{P}_{\mathrm{NBI} 1}=800 \mathrm{~kW}$ (Co-injection)
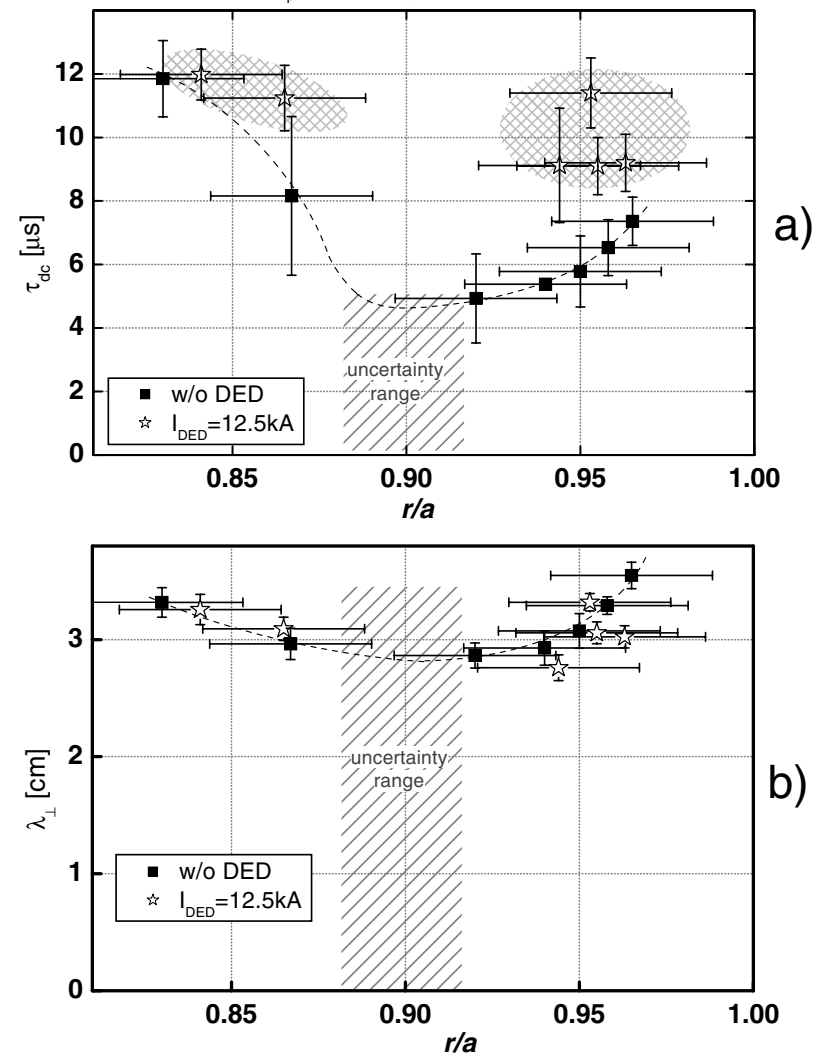

Figure 17. Turbulence decorrelation time $(a)$ and perpendicular wave length $(b)$ for DED in the $12 / 4$ configuration and non-DED operation. No data are found for $0.88 \leqslant r / a \leqslant 0.93$ due to the low plasma rotation. This region is shown by the hatched rectangle. Dashed lines give the approximate trends of data. With DED application $\tau_{\mathrm{dc}}$ increases by $\approx 35 \%$ for radii $r / a \geqslant 0.94$.

plasmas an ergodic divertor is generated in the periphery region $0.9<r / a \leqslant 1$. In this divertor region $\Omega_{\text {turb }}$ is in the idd direction. The radial extension of the ergodic divertor can be estimated from the region where the turbulence rotation is influenced by the DED $(0.9 \leqslant r / a \leqslant 0.98)$. The inner boundary of this region is an estimate of the radius of the last closed flux surface (LCFS). Good agreement is found compared with the LCFS from the field line tracing [32] for the DED configuration under consideration [19]. This demonstrates the capability of the reflectomery at TEXTOR quite well, since it is the only diagnostic from which the radial extent of the divertor and the position of the LCFS can be experimentally deduced.

Accordingly, the change in rotation to the idd direction yields that the electric field becomes positive. This means that the inversion point is shifted inward.

The decorrelation time $\tau_{\mathrm{dc}}$ of density fluctuations for plasmas with and without DED is presented in figure 17. In the region where the turbulence rotation is supposed to be small (shown by the crosshatched rectangle) no delay measurements are available. The frequency range used for the analysis is $1 \leqslant f \leqslant 75 \mathrm{kHz}$. Without the DED $\tau_{\mathrm{dc}}(r / a)$ changes from 5 to $12 \mu \mathrm{s}$ in the range $r / a=0.83-0.97$ showing a paraboliclike dependence with a minimum at $r / a \approx 0.92$. The decrease in $\tau_{\mathrm{dc}}$ is to be expected in the presence of rotation shear, as 
TEXTOR. DED in 12/4. $\mathrm{I}_{\mathrm{p}}=370 \mathrm{kA}, \mathrm{B}_{\mathrm{t}}=1.9 \mathrm{~T}, \mathrm{P}_{\mathrm{NB} 11}=800 \mathrm{~kW}$ (Co-injection)

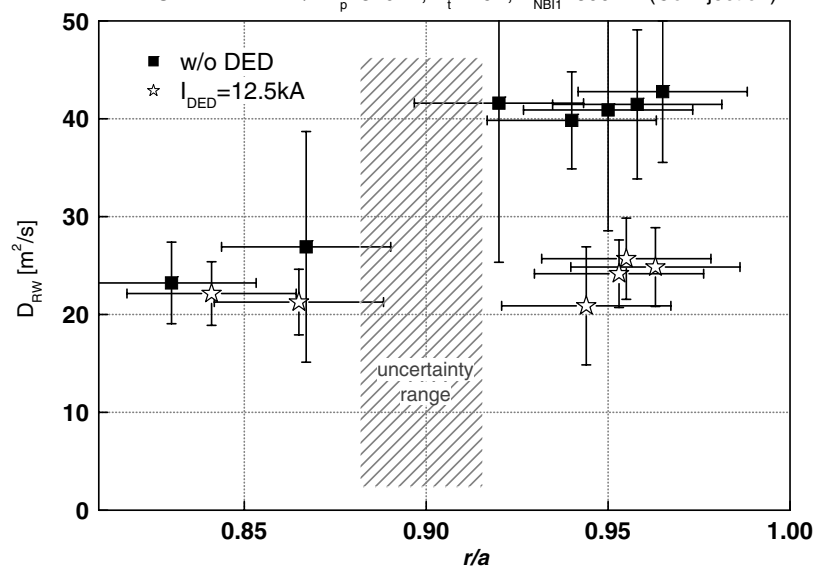

Figure 18. Random walk turbulent diffusion for Scenario 3 with and without DED operation. A local decrease of $\approx 40 \%$ in $D_{\mathrm{RW}}$ for $0.94 \leqslant r / a \leqslant 0.97$ is observed. No change in $D_{\mathrm{RW}}$ is found for $r / a \leqslant 0.88$. The hatched rectangle is the area where the low plasma rotation does not allow the estimation of $D_{\mathrm{RW}}$.

shown in figure 16. The shear tends to destroy the fluctuations, and as a consequence, the turbulence lifetime decreases. For $r / a \geqslant 0.90$ an increase in $\tau_{\mathrm{dc}}$ of $\approx 60 \%$ is found. Further inside $(r / a \leqslant 0.85)$ the effect in $\tau_{\mathrm{dc}}$ due to the DED is negligible. The time averaged perpendicular wavelength, both with and without DED, shows a weak dependence on the radius only. The values range from 0.028 to $0.036 \mathrm{~m}$ within the radial range $0.83 \leqslant r / a \leqslant 0.97$ (see figure 17).

The turbulence random walk diffusion $D_{\mathrm{RW}}$ is estimated on the basis of $\tau_{\mathrm{dc}}$ and $\left\langle\lambda_{\perp}\right\rangle$. When the DED is applied, it leads to a $40 \%$ decrease in the turbulence diffusion for $r / a \geqslant 0.9$ (see figure 18). For $r / a \leqslant 0.87$, the transport coefficient is constant and, moreover, $D_{\mathrm{RW}}$ is equal to those values obtained without DED. As a result the $D_{\mathrm{RW}}$ profile tends to be flat during the DED operation.

\section{Summary}

With the recent upgrade of the reflectometry system at TEXTOR the number of receiving horns has been increased. The system is used for turbulence investigations in the gradient as well as the periphery regions of plasma. The presence of four receiving antennas, as well as their special configuration, improves the accuracy of the turbulence propagation measurements and results in further progress in the study of the correlation properties of plasma fluctuations and its rotation.

The simultaneous measurement of toroidal, poloidal and turbulence velocity makes the comparison between plasma and turbulence velocity possible. For different radii and different toroidal plasma rotations in co- and counter-current directions, both, the LF and QC modes are frozen in the plasma. Within the accuracy of the measurements, an additional phase velocity of the turbulence, with respect to the plasma velocity, can be neglected. This offers the possibility of deriving the radial electric field from the measurement of the turbulence rotation.

The DED is a tool which influences the plasma turbulence at the edge. The applied external magnetic field breaks up the magnetic field line structure and causes an ergodization of the plasma edge.

One important effect of the DED is the modification of the radial electric field. The ergodization of the magnetic field lines leads to an increased electron loss rate which charges the plasma edge more positively. The application of the DED increases the rotation in the scrape-off layer, where the original rotation is in the ion diamagnetic drift direction. Since the rotation for $r \leqslant r_{\lim }$ is in the electron diamagnetic drift direction, the DED brakes the rotation. The inversion point of the radial electric field (as well as the poloidal rotation velocity) is shifted further inside. This effect does not depend on the DED configuration $(3 / 1$ or $12 / 4)$ but on the field strength of the perturbation field. Note that this conclusion concerns only dc DED operation; the ac DED scenarios are beyond the scope of this paper and are the subject of future work.

The combination of the DED and tangential neutral beam injection, which influence the central plasma rotation, can affect the plasma confinement. The weak neutral beam cocurrent injection (Scenario 1) results in a flat toroidal plasma rotation profile. This configuration is very unstable from the point of view of MHD and the braking effect of the DED leads to $m / n=2 / 1$ mode locking and a confinement degradation. The turbulence rotation changes to the ion diamagnetic drift direction at the $q=3$ surface and implies a change of $E_{r}$ as well. The measured turbulence characteristics in this scenario depend on the local position of measurements with respect to the locked mode. The X-point of the locked mode is close to the equatorial plane in the same toroidal section where the reflectometry is installed and, therefore, the influence of the distortion of plasma profiles can be neglected. It was shown that the shear of the rotation around $q=3$ surface results in a suppression of the QC mode and also changes the properties of the LF mode of fluctuations.

For plasma with a large toroidal rotation in the countercurrent direction no locked mode is generated due to the DED. In this case, the DED generates a transport barrier at the plasma edge $0.8 \leqslant r / a \leqslant 0.9$, which yields a $15 \%$ increasing diamagnetic energy. The turbulence rotation is decreased at the barrier, which again demonstrates the braking effect of the DED. The acceleration of rotation by counter-neutral beam injection and braking by the DED yields an increase in the velocity shear at $r / a=0.9$. At the barrier, the level of density fluctuations is constant, the turbulence decorrelation time is increased and the turbulence wavelength is decreased. The evaluation of turbulent diffusion using a random walk model yields the reduction of transport by $\approx 50 \%$ within the barrier. This is a demonstration of the DED as a tool for generation of an internal transport barrier in plasmas with counter-current neutral beam injection.

The radial range of the perturbation field of the DED in the $12 / 4$ configuration is shorter than in the $3 / 1$ configuration. The turbulence rotation measured in Scenario 3 shows the same general braking effect as discussed above. The difference compared with Scenario 1 is the shift of the plasma column to the HFS and strong neutral beam injection in the cocurrent direction before the DED application. The co-current injection changes the plasma rotation to the ion diamagnetic drift direction at $r / a \approx 0.9$, whereas the plasma boundary $0.9<r / a<1$ still rotates in the electron diamagnetic 
drift direction. Assuming that the rotation profile has two inversion points (the second one should be near $r / a=1$ ), the application of DED changes the rotation profile from the electron diamagnetic drift direction for $0.92<r / a<0.97$ to the ion diamagnetic drift direction. Thereby the assumed inversion point near $r / a=1$ vanishes and rotation shear at $r / a \approx 0.9$ is reduced.

The evaluation of turbulent transport using the randomwalk model shows a decrease of the diffusion coefficient in $0.94<r / a<0.97$ by $\approx 40 \%$. At the same time, the most interesting region around $r / a=0.9 \pm 0.1$ is left uninvestigated because the plasma rotation is too small for correlation and delay analyses. In this sense, the above local reduction in turbulent transport does not elucidate the global confinement behaviour because the expected reduction in the shear of the rotation at $r / a=0.9 \pm 0.1$ can also affect the transport.

\section{Acknowledgments}

The authors would like to thank V. Vershkov for valuable discussions. This work is supported by INTAS 2001-2056 grants.

\section{References}

[1] Vershkov V.A. et al 1999 Nucl. Fusion 391775

[2] Conway G.D. et al 2000 Phys. Rev. Lett. 84 1463-6

[3] McKee G.R. et al 2000 Phys. Rev. Lett. 84 1922-5

[4] Fonck R.J. et al 1992 Plasma Phys. Control. Fusion 34 1993-9

[5] Vershkov V.A. et al 2005 Nucl. Fusion 45 S203-26

[6] Romanelli M. et al 2006 Nucl. Fusion, 46 412-8
[7] Kraemer-Flecken A. et al 2004 Nucl. Fusion 44 1143-57

[8] Foster M., Wooton A. and McCool S. 1995 Nucl. Fusion 35 329

[9] Ghendrih P. et al 2002 Nucl. Fusion 421221

[10] Finken K.H. 1997 Fusion Eng. Des. 37335

[11] Wolf R.C.et al 2005 Nucl. Fusion 45 1700-7

[12] Finken K.H. et al 2005 Phys. Rev. Lett. 94015003

[13] Payan J. et al 1995 Nucl. Fusion 351357

[14] Uhlemann R. and Ongena J. 1999 Fusion Technol. 3542

[15] Koslowski et al 2004 31st EPS Conf. on Controlled Fusion and Plasma Physics (London, UK, 2004) vol 28G P-1.124

[16] Liang Y. et al 2004 31st EPS Conf. on Controlled Fusion and Plasma Physics (London, UK, 2004) vol 28G P-1.126

[17] Bock M. de et al 200431 st EPS Conf. on Controlled Fusion and Plasma Physics (London, UK, 2004) vol 28G P-1.117

[18] Jakubowski M. et al 2006 Phys. Rev. Lett. 96035004

[19] Lehnen M. et al 2005 Plasma Phys. Control. Fusion 47 B237-48

[20] Unterberg B. et al 2004 20th IAEA Fusion Energy Conf. (Vilamoura, Portugal, 1-6 November 2004) EX/P5-33

[21] Jakubowski M.W. et al 2004 Nucl. Fusion 44 S1

[22] Vershkov V.A. et al 1997 J. Nucl. Mater. 241-243 873-7

[23] Busch C. et al 2005 32nd EPS Conf. on Controlled Fusion and Plasma Physics (Tarragona, Spain, 2005) vol 29G P-1.016

[24] Koslowski H.R. et al 1997 Plasma Phys. Control. Fusion 39 B325-36

[25] Hazeltine R.D. 1974 Phys. Fluids 17961

[26] Liewer P.C. 1985 Nucl. Fusion 25543

[27] Greenfield C.M. et al 2000 Phys. of Plasmas 71959

[28] Doyle E.J. et al 2002 Nucl. Fusion 42 333-9

[29] Askinazi L.G. et al 2004 Plasma Phys. Control. Fusion, 44 A51-9

[30] Wolf R.C. 2003 Plasma Phys. Control. Fusion, 45 R1-91

[31] Burrell K.H. 1995 Phys. Plasmas 41499

[32] Abdullaev S.S. et al 1999 Phys. Plasmas 6135 